\title{
Sex-specific outcomes in PVI for PAD
}

Peripheral artery disease (PAD) is commonly treated using percutaneous peripheral intervention (PVI) of the lower extremities. A new paper published in $J A C C$ describes the sex-specific differences in patients undergoing PVI for PAD.

"A number of studies have reported gender differences in outcome for patients with cardiovascular disease," says P. Michael Grossman of the University of Michigan, MI, USA, who led the study. The team used the Blue Cross Blue Shield of Michigan Cardiovascular Consortium PVI registry, which included data from 12,379 patients from 16 hospitals who underwent PVI for PAD. Of this population, 5,105 (41.2\%) were female, and who were more likely to have complex and severe disease than their male counterparts.

Surprisingly, despite a higher rate of complications in women compared with men (bleeding and vascular access complications in particular), the procedural success of lower extremity PVI was roughly equal between the two sexes $(79.7 \%$ versus $81.7 \%$; $P=0.08)$. This parity was explained by the higher technical success of the procedure in women over men (91.2\% compared with $89.1 \% ; P=0.014)$. The overall major end points (in-hospital death, MACE, myocardial infarction and stroke) were similar between the two groups. "These data suggest that percutaneous revascularization is a viable alternative for women, particularly if open surgery is not an option," explains Grossman.

However, improvements can still be made. "We need to learn more about the reasons for increased risk of bleeding and need for transfusion in women, and work to decrease those complications," concludes Grossman.

Tim Geach

Original article Jackson, E. A. et al. Impact of gender on morbidity and mortality rates following lower extremity interventions for peripheral arterial disease: observations from the Blue Cross Blue Shield of Michigan Cardiovascular Consortium. J. Am. Coll. Cardiol. doi:10.1016/j.jacc.2014.03.036 